\title{
SINDICATOS Y CORPORATIVISMO EN BRASIL
}

\author{
Arthur Bragança de Vasconcellos Weintraub* \\ Universidad Federal de São Paulo, Brasil
}

\section{INTRODUCCIÓN}

En la actualidad, la legislación laboral en Brasil se basa pertinentemente en el sistema de relaciones laborales de Mussolini y en una de sus más famosas piezas de legislación: la "Carta del Lavoro” de 1927 (Carta del Trabajo). Esta legislación puede considerarse como el preámbulo de la ley italiana sobre las corporaciones.

La declaración III de la "Carta del Lavoro" estipulaba la libertad de organización sindical. Pero por el contrario, tal como se formula enseguida, sólo el sindicato legalmente reconocido por el Estado tiene el derecho de acuerdo, y por fuerza de la ley representa a toda la categoría de los trabajadores o de los empleadores para los cuales se constituye. Los sindicatos eran más una cuestión pública y no una circunstancia privada.

Brasil, vale la pena señalar, no sólo ha emulado la "Carta del Lavoro" en su Carta de Trabajo de 1943, sino que también introdujo el marco para el trabajo y empleo dentro de la jurisdicción especializada en los tribunales laborales. Es importante destacar que una de las cuestiones jurídicas más controvertidas dentro de la jurisdicción laboral y el Tribunal Superior del Trabajo de Brasil es de su jurisdicción en los casos que presentan rasgos no-civiles relativos a la relación empleado/ empleador.

La llamada consolidación de la legislación laboral (Consolidação das Leis do Trabalho-Decreto-Lei No 5.452) fue promulgada en mayo 1 de 1943. No es una coincidencia, el Día del Trabajo.

Hasta el momento, esta legislación aún esboza con amplitud todas las relaciones laborales y de empleo. Prácticamente no hay aspecto de la relación laboral que queda fuera del control legal, dadas las enormes complejidades de las normas y reglamentos. Casi ninguna negociación resulta legalmente permitida ya que el principio básico de la Ley del Trabajo y Empleo de Brasil consiste en la presunción de que el empleado hace caso omiso de sus derechos, y no puede igualmente negociar con el empleador dominante. Casi todas las iniciativas para crear una nueva situación jurídica en este ámbito fueron borrados por razones políticas permeadas por la doctrina corporativista. Obviamente, la protección social es necesaria, los derechos laborales deben ser preservados, pero es evidente que el conflicto de intereses en el contexto de los sindicatos está obstruyendo cambios en la ley.

El principal argumento sofista, hasta la fecha, para evitar cualquier alteración en el campo evaluado es que cualquier reforma legal en el contexto del trabajo o empleo, sin duda, viene a mitigar o reducir los derechos fundamentales de los trabajadores de clase, a pesar de que más de la mitad de esa clase permanece en el ámbito del sector de empleo informal. Por lo tanto, la legislación en la práctica no proporciona un nivel adecuado de seguridad y empleo para más de la mitad de los trabajadores.

\footnotetext{
Profesor de Derecho Previsional de la Universidad Federal de São Paulo - Unifesp. Coordinador de la Escuela de Ciencia Actuarial de la Unifesp. Investigador invitado de Harvard.
} 
Sin embargo, algunos sindicalistas en Brasil siguen mostrando un comportamiento corporativista sólido, propugnando el mantenimiento del Código de 1943 sobre la argumentación de que eso proporciona una protección eficaz de los trabajadores contra la arbitrariedad del empleador. Para aquellos que tienen el privilegio de tener contratos formales de empleo (sector formal) puede ser cierto. Pero el fundamento real de muchos de los sindicatos es el mantenimiento del status quo. Es patente que el ambiente de trabajo ha cambiado desde 1943. Y recordemos que esta legislación era precisa una emulación de la carta italiana de 1927. No hay dudas sobre la tendencia explotadora en la figura del empleador. Pero eso no significa una obliteración de ideas contrarias a cualquier reforma legal o constitucional.

No obstante, Brasil no ha ratificado la Convención 87 de la Organización Internacional del Trabajo (libertad de asociación y protección del derecho de sindicación). Las fuerzas sindicales internas son considerablemente más robustas con el fin de fundamentar la evitación de la ratificación. Además de eso, incluso Argentina, vecino de Brasil, que es famoso por la falta de libertad durante ciertos períodos de tiempo de la historia, ratificó el Convenio 87.

La disminución o incluso la falta de representación de los trabajadores no impiden que algunos sindicatos brasileños vean la disminución de sus asociados miembros. Eso porque la ley impone la sindicalización. El sindicalismo, como fenómeno global, por motivos diversos (Vg. ambientes de trabajo ya no propician aglutinación de personas como en tiempos industriales de decenios pasados), está en declive. Esto se debe también a que algunos sindicatos ineficaces que en ningún caso representa a los intereses de los trabajadores sindicalizados sobreviven solo de la dependencia del impuesto sindical obligatorio (salario de 1 día de cada año, los cuales el 20\% se destina al gobierno, como se expone más adelante) que se debe pagar hasta por los trabajadores no sindicalizados que enlazan con la categoría laboral. Y esta relación entre los trabajadores sindicalizados y la unión es casi obligatoria también. Los trabajadores sindicalizados no están representados con frecuencia en absoluto. Aún no sindicalizados, los trabajadores tienen que pagar el impuesto obligatorio, y no hay un marco jurídico actual para exceptuar a quien paga por sus cuotas sindicales, así, incluso si uno no tiene un Óbolo para Caronte.

Bajo este punto de vista, los empleados y los empleadores deben aceptar el hecho de que muchas veces la representación de las organizaciones sindicales y laborales, protegida por el corporativismo, puede actuar como un brazo del Estado, bajo la coordinación de los movimientos políticos. Impuesto sindical obligatorio es un ejemplo de las consecuencias en lo que respecta a un fuerte movimiento de abstención al cambio en la política laboral, que subvenciona los sindicatos que no necesariamente protegen a los trabajadores representados.

El propio Estado retiene los impuestos que en un principio se justifican sobre la base de los intereses obreros. La duración del Fondo de Garantía del Tiempo de Servicio (FGTS-Fundo de Garantia do Tempo de Serviço): empleadores hacen depósito de 8,5\% de la remuneración en cuenta conjunta en un Banco Federal específico (Caja Económica Federal), el balance publicado, siempre y cuando el trabajador es despedido sin causa, o por motivo de SIDA, cáncer, o para la compra de una casa, por ejemplo. En la práctica, la tasa de interés del gobierno de ese ahorro de inversión es generalmente de $3 \%$ sobre una base anual, que históricamente en Brasil está muy por debajo de la tasa de inflación. Cualquier otra inversión de bajo perfil puede acumular mucho más que eso.

La Constitución brasileña de 1988 es el vivo ejemplo de la complejidad de la influencia italiana. Los artículos 7 y 8 son meticulosamente intrincados en el sentido de que una serie de derechos de los trabajadores (artículo 7) y elementos del sindicalismo (artículo 8) se determinan perentorios. Los derechos fundamentales del trabajo, que son derechos fundamentales 
introducidos en la Constitución, garantizan los requisitos mínimos de protección, pero las complejidades indican que los intereses corporativos están en juego en la medida en que los legisladores constitucionales centran en detalles tales como derechos de los trabajadores domésticos.

La voluntad del legislador Brasileño constitucional -menslegislatoris- en 1988 fue impulsada profusamente por un corporativismo nativo inherente. Las razones expuestas anteriormente explican la falta de flexibilidad en relación al mercado de trabajo brasileño. El ámbito jurídico laboral es sin duda excesivamente regulado y la negociación privada se evita sistemáticamente por un discurso impreciso en lo cual el empleado se considera un ser incapaz que no puede disponer de su propio empleo o derechos conexos al trabajo humano. Pera reformar este sistema, es lo más de las veces tachado como intentarse un daño a los derechos de los trabajadores. Un dogma.

A pesar de que la Constitución permite a los empleadores y trabajadores se pongan de acuerdo sobre los salarios, las prestaciones sociales se interpretan legalmente como derechos fundamentales de los trabajadores para negociar. Es un error aceptar la explotación de los empresarios, pero hay muchos aspectos en el campo laboral en el sistema legal de Brasil para ser actualizados. Es importante hacer hincapié en que la protección social puede existir incluso si se implementan los cambios mencionados anteriormente, especialmente llevado a la vista el mercado informal y la inclusión social por la formalización de los trabajadores.

\section{CORPORATIVISMO}

En principio, el corporativismo (derivada del latín corpus, que literalmente significa cuerpo) puede implicar la posibilidad de que la clase trabajadora se organice teniendo la ventaja del poder de la colectividad. Por una parte se puede aumentar la mano de obra y la protección del empleo. Por otro lado, puede convertirse en un instrumento para algunos grupos determinados de la sociedad para manipular la política local o nacional. En ese sentido, estos grupos dependen de la intervención del Estado.

El modelo de Estado corporativista proviene de raíces italianas. Getulio Vargas, el dictador brasileño por años y gobernante democrático después, inspirado por el Duce Mussolini, fue el actor más importante responsable por la introducción sólida del corporativismo en Brasil. Siguiendo la metodología del dictador italiano, Vargas, quien se desempeñó como presidente desde 1930 a 1945 y desde 1951 hasta que se suicidó en 1954, trató de centralizar todo el poder en el gobierno, donde se ejercía el control absoluto. La cooptación de los sindicatos y organizaciones laborales fue esencial en este aspecto, y la supresión de los movimientos de izquierda en ese período de tiempo era crucial.

Además, la importancia de centralizar el poder del gobierno, la mediación de las fuerzas del mercado y evitar las ideas revolucionarias contra el sistema corporativo fue enorme. La estructura sindical construida durante el gobierno de Vargas estaba profundamente consolidada bajo la influencia del corporativismo. El Estado debe intervenir difusamente en la sociedad: exactamente el modelo italiano de la época.

Massoni (2007) examina el corporativismo como un:

"Fenómeno dentro de la sociedad basada en los principios de organización del Estado, a fin de mitigar los conflictos. Saldrá a la dirección opuesta al individualismo, con el alcance de inculcar gradualmente la solidaridad en la sociedad. En este aspecto, el aislamiento de los individuos en la sociedad, como la doctrina liberal del siglo XIX se indica, es una "caracte- 
Arthur Braganca de Vasconcellos Weintraub / Sindicatos y corporativismo en Brasil

rística de la sociedad que debe evitarse". La doctrina liberal, como una línea de pensamiento individualista, clasifica a la persona al mismo tiempo como el origen y el fin último del Estado" (énfasis añadido).

La paz social y la productividad dentro de la intervención del Estado nacional es justificada en zonas ampliamente distribuidas como en las organizaciones laborales. El paradigma fascista italiano asignó sindicatos como órganos auxiliares del Estado. No se interprete la representación sindical como sencilla negociación colectiva privada. Que el rango de la función de los sindicatos se convirtió en una cuestión de derechos públicos. Bajo este punto de vista, el corporativismo esencialmente asigna la representación sindical como una representación institucional.

Así, en referencia a esa doctrina, el Estado no es un natural intermediario entre grupos internos y poderes. El Estado es el órgano superior intrínseco al sistema social. Controla y preserva el tejido social, estimulando la armonía social y el desarrollo, todo ello bajo el pretexto del nacionalismo. Programas corporativos fascistas fueron diseñados para servir a algunos objetivos importantes, como el crecimiento industrial.

En Manoilesco (1938), “corporativismo hace hincapié en que la colectividad nacional representa una entidad superior con una marcada personalidad a distinguir atributos en relación con las personas. El Estado no es neutro o agnóstico, sino algo más bien ideal y superior en relación a las personas que pueden ser consideradas instrumentos al servicio del Estado".

El régimen corporativo comprende las personas, los grupos de la sociedad y el propio Estado. El sistema político, se ha dicho, asigna varios grupos diferentes, por ejemplo, mano de obra, el gobierno y la industria, organizados de manera corporativa.

A la luz del conjunto expuesto anteriormente, el corporativismo abarca sindicatos también. Las organizaciones sindicales son agentes de facto del Estado en ese sentido. Mortati (1931) examina el régimen corporativista bajo el punto de vista de una:

"nueva organización de las clases productivas en las que el objetivo principal es "prevenir o mitigar los conflictos con la autoridad del Estado" si es necesaria. El Estado coordina todas las actividades productivas en los grupos sociales con el fin de maximizar la productividad mientras que toda esta organización tiene como objetivo el ámbito del Estado" (énfasis añadido).

El corporativismo no determina el Estado y los ciudadanos como antípodas. Muy por el contrario, esta doctrina citada propugna superioridad jerárquica de los objetivos del Estado en relación a la intención de los individuos.

De hecho, el sistema corporativo en sus primeros tiempos se suponía sustituir la economía liberal, impregnada de libre mercado -laissez-faire-, invocando criterios de economía corporativa en la que los intereses privados son relevantes sólo en su función auxiliar en relación con el Estado. En teoría, el Estado establece las directrices nacionales sosteniendo firmemente un exclusivo objetivo: bien común.

Al menos en teoría, el corporativismo evoca una obstrucción de competencias desleales entre los individuos y grupos en la sociedad. La razón fundamental es la igualdad. Conflicto entre el capital y el trabajo estaba siendo ignorado bajo la doctrina liberal de libre mercado. El Estado no corporativista ignora la fricción que el capital/relación de trabajo podrían ocasionar. Un ideal 
de la igualdad y una libertad generalizada aparente bajo el imperio de la ley llevó a las circunstancias en las que la desigualdad y la iniquidad engendraron desequilibrio de condiciones entre las personas. Para empeorar la situación, la clase de fuerza de trabajo no se organizó lo suficiente para hacer frente a los grupos de poder que podrían tomar ventaja de este supuesto desequilibrio social. El corporativismo surge por lo general como una panacea para todos los problemas originados después de la Revolución Industrial y durante la evolución de las primeras etapas del capitalismo en el primer cuarto del siglo XX.

Giannotti (1987) afirma que el verdadero papel de los sindicatos en este contexto es proteger la interrelación y cooperación entre el trabajo, el capital y el gobierno.

\section{ORDENAMIENTO JURÍDICO CORPORATIVISTA EN ITALIA}

El factor principal de la doctrina corporativista es la organización de las fuerzas sociales que mezclan los sectores privados en un estilo de corporativismo público. En Italia, al 03 de abril 1927, la Ley Rocco fue promulgada para disciplinar las relaciones colectivas de trabajo y se componía de tres capítulos:

1.- La administración legal de los sindicatos

2.- Tribunales y jueces laborales

3.- Bloqueo y la huelga

En el mismo año se ha crea la regulación de la ley. Todo este aumento de la legislación era más que una mera construcción de un sistema jurídico privado, era uno de los principales pilares del nuevo sistema social, es decir, el sistema jurídico corporativista.

Es importante destacar que la concepción básica del corporativismo parece estructurada para servir a varios propósitos principales, y uno de ellos es la protección de los empleados contra los abusos que podrían ser perpetrados por los empleadores. La vulnerabilidad a la explotación lograría ser suprimida en teoría por la intervención del Estado.

La Ley Rocco fue promulgada bajo las directrices del Consejo fascistas cual norma básica primaria. Era la mano de obra pura de las organizaciones de control por parte del Estado.

Tal como está formulada en la Ley Rocco, un solo sindicato está autorizado para representar a cada categoría de trabajadores. Y cada sindicato representaba no sólo a los empleados sindicalizados, porque recibieron la cohorte de poder de establecer convenios y acuerdos colectivos vinculantes para toda la categoría.

Rosado (1937) afirmó que la Ley del Trabajo y los Tribunales de la Ley de Empleo fueron los responsables de hacer cumplir los convenios colectivos antes mencionados. La creación de estos tribunales especializados prohibió oficialmente la defensa de cada categoría.

En esencia, la huelga y el cierre patronal era ilegal en virtud de las características anti-económicas y antisociales que se consideraron antinacionales. Huelga y cierre patronal eran delitos.

En Italia, después de la promulgación de la Constitución de 1947, se requieren algunos aspectos formales en relación con la creación de sindicatos.

Según Galantino (2000), la Constitución italiana permite la libertad privada a la luz de la organización del trabajo y sobre el alcance de la acción de los sindicatos. 


\section{EL CORPORATIVISMO Y LAS ORGANIZACIONES LABORALES EN BRASIL}

Para Romita (1976), la legislación que abarca sindicatos brasileños poco después de la creación de la República podría disfrutar de un cierto grado de libertad en relación con la pluralidad y la representación. Sin embargo, en vista de que, históricamente, Brasil era básicamente un país agrícola, el marco de las organizaciones obreras era todavía muy débil en el comienzo del siglo XX. A pesar de algunas manifestaciones de los trabajadores organizados, tales como huelgas y aglutinación de trabajadores, los sindicatos no evolucionaron.

En relación con su marco histórico del mercado de trabajo, hasta el 13 de mayo 1888 la esclavitud en Brasil era legal. El país se asienta casi enteramente en el trabajo rural. El sistema de plantaciones de café dependía de trabajo esclavo.

Después de la abolición de la esclavitud, la mano de obra que vino a sustituir el trabajo que los esclavos estaban realizando era europea, sobre todo de Italia. Y los trabajadores italianos han traído las ideas básicas de la organización sindical y trabajo que se ha extendido ampliamente en el viejo continente.

En la primera Constitución de la República de Brasil, promulgada en 1891, los derechos de asociación establecidos en ese sentido de la apertura a nuevas ideas vienen con los ideales que los inmigrantes traían de Europa. El Decreto 979, promulgado el 6 de enero de 1903, permitió a los profesionales de la agricultura y la industria rural organizarse por medio de los sindicatos con el fin de proteger sus intereses.

El predominio rural absoluto del país repercutió en el contenido de la legislación. A pesar del hecho de que la ley ha dado lugar a la creación de sindicatos rurales, en virtud de lo primitivo de las condiciones generales de trabajo, el incipiente movimiento de mano de obra rural no encontró un terreno fecundo para desarrollarse.

Sin embargo, el país estaba experimentando alguna industrialización incidental. En 1907, la Ley 1.637 se promulgó en términos de dar la posibilidad a los trabajadores en cualquier tipo de profesión organizarse con la libertad de asociación y con el alcance de la protección laboral.

La estructura sindical incipiente que ha prosperado no fue lo suficientemente fuerte para sobrevivir a la revolución que dio lugar Vargas en 1930. Es relevante destacar que en noviembre de ese mismo año Vargas creó el Ministerio de Trabajo, que es un instrumental fundamental en términos de control del Estado sobre aspectos laborales generales de la nación. Regulación específica (Decreto 19.770) promulgada en 1931 rescindió la autonomía a los sindicatos para someterlos al poder del Estado. A partir de entonces, los sindicatos eran meras entidades auxiliares del gobierno a través de las cámaras de conciliación y juicios que regulan los conflictos entre empleadores y empleados, entre otras funciones. Esencialmente, la regulación puede ser considerado como pilares para la creación de un modelo sindical corporativista en Brasil.

Decreto 19.770: en 19 de marzo 1931 este decreto creó las normas de la Asociación Sindical. El sindicato se define como un órgano de colaboración en "unicidad" (sin pluralismo). Hay una prohibición de todo trato con entidades internacionales y hay hipótesis de la intervención del Estado en la organización del trabajo. El Estado delega funciones públicas a los sindicatos, como la aplicación de los derechos y las agencias administran el empleo, las entidades cooperativas, servicios de salud, escuelas y otras entidades filantrópicas.

El decreto antes descrito determinó que cada rama de la actividad laboral debe ser comprimida en una categoría por cada sindicato. La categoría comprende los trabajadores que ejercen 
profesiones idénticas, similares o entrelazadas. Esta medida restringe la libertad de determinar el marco de la Unión en el ámbito profesional.

Para Joaquim Pimenta (1957), unicidad (prohibición geográfica de más de un sindicato en cada municipio -que todavía existe en Brasil hasta la fecha), que representa la categoría como su órgano exclusivo, es un dispositivo esencial para la eficaz cooperación entre el sindicato y el gobierno.

Unicidad consiste en un dispositivo de evidente control sobre los sindicatos. Esto es así, ya que facilita el control del Estado sobre los sindicatos e inhibe por medio de la ley la creación de la competencia entre los sindicatos que podrían generar un desarrollo natural de la estructura de organización del trabajo. Y cualquier novedad en cuanto a la eficiencia y la complejidad podrían obstaculizar el ámbito sindical controlado por el Estado.

El sindicato corporativista es el producto emblemático de la política específica de Vargas durante la década de 1930, en que por Gomes (1999), la inserción de los sindicatos en la maquinaria del Estado y en el cuerpo de leyes era una premisa subyacente.

En realidad, Gomes (op. cit.) es muy profundo al decir que el Estado autoritario, domina sindicatos. Esta afirmación no significa que los sindicatos fueron neutralizados o erradicados. Para Gomes, el Estado "absorbe" los sindicatos y, además de eso, los sindicatos transformasen en una ramificación de Estado sobre la fuerza de trabajo y ámbitos profesionales. Era "el fin de la sindicalización real".

Algunos derechos laborales fueron borrados durante el interregno en el que el Estado brasileño tiene el poder de controlar los aspectos principales de la maquinaria y las funciones de los sindicatos. La libertad de asociación no existía y el derecho de asociación no fue ejercido voluntariamente.

Cabe destacar que la Constitución de 1934 restableció formalmente la autonomía y la pluralidad en el ámbito de la Unión. De hecho, hubo una revolución que ocurrió en el estado de São Paulo contra la dictadura y el dominio de Vargas en todo el país. São Paulo perdió la guerra, después de numerosas muertes, pero se las arregló para forzar a Vargas a la promulgación de la nueva Constitución. Esa es la razón por la que algunos aspectos de la nueva Carta Magna eran más que un método formal de la legislación con el fin de componer la insurgencia.

En la práctica, el Gobierno podría formular decretos con fuerza de ley. Por lo tanto, la regulación relativa a las relaciones sindicales y los sindicatos corporativistas guardaba todos los rígidos principios anteriores a la Constitución de 1934.

En noviembre de 1937, Vargas derribó toda la oposición en unos movimientos políticos y militares que siguieron el llamado "Nuevo Estado"; con la creación de una nueva Constitución en la que todo el ámbito sindical y laboral y el contexto de organizaciones eran casi literalmente una emulación de la fascista "Carta del Lavoro".

A pesar de que la carta constitucional deja libertad profesional, de asociación y la libertad en materia de sindicalización, el control gubernamental y la influencia sobre la estructura interna de los sindicatos se convirtió en draconiana. La posición paternalista del Estado se hizo tan fuerte (y no sólo en el concepto de mano de obra) que hasta la fecha parece ser notablemente indeleble.

La Constitución de 1937 presentó las líneas generales de los sindicatos y sus principales características:

1.- $\quad$ La protección de los derechos laborales y los intereses profesionales de los miembros;

2.- La organización de los derechos y obligaciones concernientes a la relación entre empresarios y trabajadores que resulten de las condiciones de una actividad económica específica 
por medio de convenios colectivos (convenios/convenciones colectivos de trabajo para categorías o acuerdos colectivos de trabajo para empresas o grupos de empresas);

3.- Colaboración con el Estado en la solución de los conflictos laborales relacionados con los intereses de los respectivos miembros.

El artículo 140 de la Constitución mencionada, determina que los medios de producción de la economía deben ser "organizados en las empresas que, como entidades representativas de las fuerzas nacionales de trabajo, están dentro de la protección del Estado". Esas "corporaciones" fueron consideradas por la Constitución como "órganos que ejercen funciones "delegadas" del poder público" (énfasis añadido).

La regulación sindical llegó a un formato más definido en 1939 por medio del decreto número 1.402, en que la exclusividad geográfica de la representación a los sindicatos es explícitamente consolidada (máximo uno por ciudad).

Este tipo de configuración de la infraestructura de la organización del trabajo elimina virtualmente cualquier forma de competencia entre los sindicatos y fue incorporado a la CLT -Consolidação das Leis do Trabalho- en 1943. Hasta la fecha sigue siendo aplicable. Todas las Constituciones posteriores en Brasil (1946, 1967, 1969 y 1988) adoptaron el mismo modelo formal asentado en la libertad de asociación como una simple formalidad, mientras que el sistema carecía de verdadera pluralidad, autonomía individual y libertad.

La consolidación de la legislación laboral-Consolidação das Leis do Trabalho, que la sigla es ampliamente conocida en Brasil como CLT, dispone en su artículo 579 que el impuesto sindical debe ser pagado por todos los miembros de una cohorte económica o profesional específica. La Constitución Federal en su artículo 149 y el código de impuestos en el tercer artículo corroboran el carácter fiscal obligatorio de la contribución. Tal contribución no depende de la voluntad del empleador o del empleado.

El resultado final de este sistema sindical que no se basa en la representación real, sino más bien en los impuestos obligatorios, es una miríada de organizaciones repartidas por todo el país. Los sindicatos se producen de forma masiva, incluso en las regiones no industrializadas. Y, además de eso, el número de sindicatos en Brasil es asombroso. En 2005, sindicatos formalmente registrados de acuerdo con el Ministerio de Trabajo alcanzaban los $23077^{1}$.

La Constitución Federal brasileña en el artículo 8 establece la libertad de asociación, profesional o en materia de sindicalización. Sin embargo, la sección (incisión) IV del mismo artículo determina que la afiliación es irrelevante en una referencia al impuesto obligatorio o contribución sindical (contribuição sindical). Los empleados, los empleados domésticos, trabajadores autónomos están obligados a pagar la contribución sindical en relación con su profesión o categoría.

Un banco federal (Caixa Econômica Federal) mantiene cuentas específicas clasificadas a cada una de las organizaciones de trabajadores que se beneficiaron de que, en aplicación de los artículos 588 y 589 de la CLT, el dinero debe ser distribuido en la siguiente proporción:

1. $5 \%$ a la Confederación correspondiente;

http://portal.mte.gov.br/data/files/FF8080812BAFFE3B012BB54E200A341F/DIAGNOSTICO_DAS_RELACOES_DE_ TRABALHO_NO_BRASIL.pdf 


\section{2. $15 \%$ a la Federación correspondiente;}

3. $60 \%$ a sindicato correspondiente;

4. $20 \%$ a la "cuenta especial de empleo y sueldo".

La "cuenta especial de empleo y sueldo" es administrado por el Ministerio de Trabajo que designa la distribución de este dinero.

En los casos en que no hay pago de las cuotas sindicales requieren ejecución judicial promovida por las organizaciones sindicales. Esta acción legal se tiene que presentar en los tribunales laborales, en virtud de jurisdicción laboral específica, una característica notable del corporativismo actual en Brasil. La enmienda constitucional número 45/2004 corrobora la competencia jurisdiccional de los tribunales laborales para juzgar cualquier litigio relacionado con la ejecución legal de los impuestos/contribuciones sindicales.

En virtud del artículo 149 de la Constitución brasileña de 1988, sólo el gobierno federal o el poder legislativo federal tienen el poder de crear las cotizaciones sociales de los intereses de las categorías profesionales o económicas, lo que significa que los estados y municipios no pueden crear impuestos o contribuciones sindicales.

Las cuotas sindicales pueden ser refutadas por los trabajadores si la organización del trabajo no ofrece ninguna ayuda o en ausencia de los miembros. En efecto, un procedimiento de este tipo llevará a un esfuerzo hercúleo por parte del trabajador. Sin embargo, es técnicamente factible.

Los empleadores tienen la obligación de las cuotas sindicales en virtud de la naturaleza inherente a los impuestos de estos pagos. Según el artículo 578 de la consolidación de la legislación laboral, el factor que genera la obligación de pagar el impuesto obligatorio es la clasificación en una categoría económica determinada.

En caso de personas jurídicas que realizan una variedad de actividades, el método para determinar la forma de clasificar la categoría económica se funda en la actividad predominante o preponderante. En general, los soportes relacionados con las contribuciones sindicales de los empleadores y las personas jurídicas varían de acuerdo al capital social.

Los sindicatos monopolizan la representación laboral en la ley. Los trabajadores pueden ser representados por un solo sindicato en cada segmento de la profesión o categoría dentro de un ámbito geográfico municipal. Por lo tanto, no hay competencia entre los sindicatos. Los sindicatos tienen cierta autonomía, pero los trabajadores no han adquirido la libertad de organización. Este modelo es una función híbrida de los sindicatos en Brasil mezclando elementos liberales y características corporativistas. Este fenómeno impide al país ratificar la Convención 87 de la Organización Internacional del Trabajo.

Después de la promulgación de la Constitución de 1988, algunos aspectos del corporativismo fueron borrados o mitigados. El derecho a la huelga y el cierre patronal son buenos ejemplos para corroborar esta afirmación. Los sindicatos comenzaron a tener más independencia para organizarse y para negociar sin la intervención del Estado, y a esto se suma otro elemento importante de la relación entre el Estado y el poder sindical. Por lo menos legalmente el gobierno no tiene autonomía para intervenir en los asuntos internos de los sindicatos.

Convenios colectivos de trabajo o convenciones colectivas de trabajo se pueden realizar entre sindicatos y empleadores. La diferencia la participación de convenciones colectivas de trabajo y los acuerdos colectivos de trabajo es básicamente: 
Arthur Braganca de Vasconcellos Weintraub / Sindicatos y corporativismo en Brasil

1.- Acuerdos colectivos de trabajo: consisten en una negociación colectiva que abarca sólo un sindicato específico y uno o más empleadores correlacionados (por ejemplo, empresa o empresas en la misma actividad económica);

2.- Convenciones colectivas de trabajo: consisten en una negociación colectiva que afecte a dos o más sindicatos y de toda la categoría de los trabajadores representados por los sindicatos.

Es importante destacar que el sistema unitario establecido anteriormente, presenta una marcada ramificación en cada sector de la estructura de los sindicatos, mitigando así la posibilidad de aglutinación en los movimientos de trabajo. Esta aglutinación podría privar a la capacidad del gobierno en términos de control de eventos sociales. No existe una entidad capaz de reunir a todos los sindicatos. Esta es una tarea para el Estado como el principal organizador de los movimientos sociales. Y este concepto se deriva del corporativismo idealizado durante el gobierno de Vargas, en el que no hay espacio para cualquier organización social fuera de la esfera de control del Estado.

Es importante destacar que existen numerosas situaciones positivas en la historia de Brasil en la que los sindicatos llevan a cabo un papel muy relevante en favor de los trabajadores. No hay duda de que, particularmente en la región del sudeste, los sindicatos han desempeñado un papel fundamental en la organización de los trabajadores.

Especialmente en el campo industrial, el papel sindical en la época de desarrollo de la década de 1970 es innegable en términos de la defensa de los intereses del distrito electoral, proteger y dar significados laborales favorables a los trabajadores. Utilizando el ejemplo presidencial, Lula ha forjado su carrera en ese glorioso momento en que Brasil fue creciendo al igual que China está creciendo en los últimos años. En las organizaciones laborales de 1980 tuvo múltiples logros a su favor. El estado actual de desarrollo de la negociación colectiva en Brasil tiene sus raíces en este contexto.

Tiempos gloriosos dieron también lugar, para que los sindicatos aumentaran la rigidez del mercado laboral. Es un hecho que hoy en día Brasil está perdiendo espacio para otras economías en el mercado mundial debido a la falta de cambio en sus estructuras de trabajo y empleo. Es indiscutible que los requisitos mínimos de protección de los trabajadores y el empleo tienen que ser irrevocable. Sin embargo, ya que prácticamente todos los aspectos de la relación laboral se definen tanto por la Constitución, la regulación de CLT, el sistema de trabajo en Brasil está restringido enormemente en términos de libertad contractual entre el empleador y el empleado.

Debido al hecho de que la propia Constitución esboza muchos elementos del contrato de trabajo, un principio laboral importante consiste en la condición inquebrantable de los derechos laborales. Y como consecuencia, los términos del contrato de trabajo se convirtieron rotundamente rígidos.

El mercado de trabajo brasileño tiene que ser adaptable. Circunstancias globales son sumamente propicias para las adaptaciones en las instituciones del mercado de trabajo y los reglamentos laborales y de empleo. Políticas públicas pobres en materia laboral, intrínsecas a un residual contexto corporativo, todavía obstaculizan el desarrollo y desfiguran la riqueza del país.

La inmutabilidad de las leyes laborales es sin duda un obstáculo para el desarrollo económico brasileño. En Brasil, la ley abarca prácticamente todos los aspectos de la relación entre el empleador y el empleado. La premisa principal es que el empleado debe ser protegido en todos los aspectos de su vida laboral y no puede decidir por sí mismo. 
Como dijo Balzac, un país con demasiadas leyes no tiene ley. Y tomado en su conjunto, los sindicatos en muchos campos no están involucrados con la protección real de su circunscripción. Por lo tanto, el Estado brasileño en un sentido resuena la idea antes mencionada de negarse a modernizar el modelo sindical, de empleo y la legislación laboral.

\section{BIBLIOGRAFÍA}

Galatino, Luisa. Diritto Sindacale. -10ª edizione-. Torino, Itália: G. Giapichelli Editore, 2000.

Giannotti, Vito. A liberdade sindical no Brasil. São Paulo, Brasiliense, 1987.

Gomes, Orlando. Curso de Direito do Trabalho. Río de Janeiro, Forense, 1999.

Manoilesco, Mihail. O século do corporativismo. Trad. Azevedo Amaral. Río de Janeiro: José Olympio Ed., 1938.

Massoni, T. O. Representatividade Sindical. -1. ed.-. São Paulo, LTr, 2007.

Mortati, Costantino. L'ordinamentodel governo nelnuovodirittopubblico italiano. Roma: Anonima Romana Editoriale, 1931.

Pimenta, Joaquim. Sociologia econômica e jurídica do trabalho. Livraria Freitas Bastos, 1957.

Romita, ArionSayão. Direito Sindical Brasileiro. Río de Janeiro, Brasília/Rio, 1976.

Rosado, João de Barros Couto. O direito do trabalho no corporativismo italiano. Lisboa, Portugal: LivrariaPortugália, 1937. 